\title{
Inflammation responses in patients with pulmonary tuberculosis in an intensive care unit
}

\author{
QIU-YUE LIU ${ }^{1}$, FEN HAN ${ }^{2}$, LI-PING PAN ${ }^{3}$, HONG-YAN JIA ${ }^{3}$, QI LI ${ }^{1}$ and ZONG-DE ZHANG ${ }^{3}$ \\ ${ }^{1}$ Department of Tuberculosis; ${ }^{2}$ Intensive Care Unit; ${ }^{3}$ Beijing Key Laboratory of \\ Drug Resistance Tuberculosis Research, Beijing Chest Hospital, Beijing Tuberculosis and \\ Thoracic Tumor Research Institute, Capital Medical University, Beijing 101149, P.R. China
}

Received September 24, 2016; Accepted August 18, 2017

DOI: $10.3892 / \mathrm{etm} .2018 .5775$

\begin{abstract}
Pulmonary tuberculosis caused by Mycobacterium tuberculosis remains a global problem. Inflammatory responses are the primary characteristics of patients with pulmonary tuberculosis in intensive care units (ICU). The aim of the present study was to investigate the clinical importance of inflammatory cells and factors for patients with pulmonary tuberculosis in ICU. A total of 124 patients with pulmonary tuberculosis in ICU were recruited for the present study. The inflammatory responses in patients with pulmonary tuberculosis in ICU were examined by changes in inflammatory cells and factors in the serum. The results indicated that serum levels of lymphocytes, plasma cells, granulocytes and monocytes were increased in patients with pulmonary tuberculosis in ICU compared with healthy controls. The serum levels of inflammatory factors interleukin (IL)-1, IL-6, IL-10, IL-12, and IL-4 were upregulated in patients with pulmonary tuberculosis in ICU. Lower plasma concentrations of IL-2, IL-15 and interferon- $\gamma$ were detected in patients with pulmonary tuberculosis compared with healthy controls. It was demonstrated that high mobility group box-1 protein expression levels were higher in the serum of patients with pulmonary tuberculosis compared with healthy controls. Notably, an imbalance of T-helper cell (Th)1/Th2 cytokines was observed in patients with pulmonary tuberculosis. Pulmonary tuberculosis caused by M. tuberculosis also upregulated expression of matrix metalloproteinase (MMP)-1 and MMP-9 in hPMCs. In conclusion, these outcomes demonstrated that inflammatory responses and inflammatory factors are associated with the progression of pulmonary tuberculosis,
\end{abstract}

Correspondence to: Professor Zong-De Zhang, Beijing Key Laboratory of Drug Resistance Tuberculosis Research, Beijing Chest Hospital, Beijing Tuberculosis and Thoracic Tumor Research Institute, Capital Medical University, 10 Xitoutiao Street, Beijing 101149, P.R. China

E-mail: zhangzongdebj@yeah.net

Key words: pulmonary tuberculosis, inflammatory responses, inflammatory factors, extracellular signal-regulated kinase/Akt suggesting that inhibition of inflammatory responses and inflammatory factors may be beneficial for the treatment of patients with pulmonary tuberculosis in ICU.

\section{Introduction}

Tuberculosis is an infectious disease caused by Mycobacterium tuberculosis or the other members of the Mycobacterium complex such as Mycobacterium africanum and Mycobacterium bovis. The disease remains a major threat to global public health and presents increasing morbidity and mortality rates worldwide $(1,2)$. There were 1.5 million cases of mortality and 9 million new cases of tuberculosis in 2013 worldwide (3). M. tuberculosis can invade numerous organs in humans, but primarily affect the lung function (4). Cough and phlegm are the most common early symptoms of tuberculosis, along with blood or blood clots in the phlegm (5). Clinically, patients with tuberculosis are admitted to intensive care units (ICU) to monitor physical symptoms and inflammation $(6,7)$. In the last decade, more efficient drug targets for tuberculosis have been explored and the pathological mechanisms of M. tuberculosis have been investigated in order to understand its pathogenesis $(8,9)$. These drugs and mechanisms of pathology of $M$. tuberculosis have contributed to clinical treatments for patients with tuberculosis $(10,11)$. Notably, the most common pathology characteristic of M. tuberculosis is inflammatory responses in patients.

Although previous reports have indicated the significance of inhibition of inflammation responses in patients with tuberculosis (12-14), the molecular mechanisms of the M. tuberculosis-induced signaling pathway are seldom reported and must be further analyzed in human pleural mesothelial cells (hPMCs). M. tuberculosis infection commonly leads to recruitment of leukocytes and formation of granulomas around the infected macrophages, which results in limitation of the spread of $M$. tuberculosis in the lungs (15). Previous work indicates that inflammation responses are host-directed therapies for patients with M. tuberculosis infection (16). Tsenova et al (17) demonstrated that inflammation accelerates pathology in a rabbit model of active pulmonary tuberculosis. Furthermore, inflammation in patients frequently induces intraocular inflammation, chronic pulmonary heart disease and other syndromes $(18,19)$. These reports suggest that 
inflammation may be a key inducer for aggravated pathology for patients with tuberculosis.

Currently, tumor necrosis factor- $\alpha$ (TNF- $\alpha$ ) and matrix metalloproteinases (MMPs) are reported to be associated with the pathological processes of tuberculosis (20). A previous study indicated that TNF- $\alpha$ expression is associated with pathogenesis and progression of patients with pulmonary tuberculosis (21). Mihaltan (22) also suggested that TNF- $\alpha$ blockers are beneficial for the treatment of pulmonary tuberculosis. In addition, MMP-1 polymorphism has been indicated as a risk factor for fibrosis after pulmonary tuberculosis (23). Furthermore, the role of MMP-8 in 5' adenosine monophosphate-activated protein kinase-dependent matrix destruction in human pulmonary tuberculosis has been studied and the results demonstrated that neutrophil-derived MMP-8 serves a key role in the pathology of tuberculosis (24). These reports suggest that MMPs and TNF- $\alpha$ are associated with the progression of tuberculosis.

In the present study, the inflammatory factors in patients with pulmonary tuberculosis were investigated. The balance of $\mathrm{T}$ helper cell (Th)1/Th2 cytokines and the expression levels of interferon (IFN)- $\gamma$, interleukin (IL)-10, IL-12, and IL-4 were analyzed. The TNF- $\alpha$ and MMP-induced extracellular-signal-regulated kinase (ERK)/Akt signaling pathways were investigated in hPMCs isolated from patients with pulmonary tuberculosis.

\section{Materials and methods}

Ethics statement. The study protocol was performed according to the Guide for the Care and Use of Clinical Patients of Capital Medical University (Beijing, China). The study was approved by the Ethics Committee of Beijing Chest Hospital (Beijing, China). Informed consent was provided by all participants. A total of 124 patients (12-58 years old, 72 male and 52 female) with M. tuberculosis infection who had been admitted to ICU were recruited to analyze inflammatory cell and factor expression in Beijing Tuberculosis and Thoracic Tumor Research Institute (Bejing, China) between May 2012 and June 2014. A further 52 healthy volunteers (21-46 years old, 32 male and 20 female) were recruited as a control group between May 2012 and May 2013.

Cell culture. hPMCs were obtained from patients and healthy volunteers and cultured in Dulbecco's modified Eagle's medium (Gibco; Thermo Fisher Scientific, Inc., Waltham, MA, USA) supplemented with $10 \%$ fetal bovine serum (Sigma-Aldrich; Merck KGaA, Darmstadt, Germany). hPMCs were cultured in a $5 \% \mathrm{CO}_{2}$ incubator with a humidified atmosphere at $37^{\circ} \mathrm{C}$.

ELISA. Blood samples $(15 \mathrm{ml})$ were collected from clinical patients and healthy volunteers via the jugular vein catheter. Serum was obtained from blood via centrifugation at $6,000 \times \mathrm{g}$ at $4^{\circ} \mathrm{C}$ for $15 \mathrm{~min}$. In the protein detection assay, human MMP-1 (DY901B), MMP-9 (DMP900), TNF- $\alpha$ (DTA00C), IL-1 (DLB50), IL-6 (D6050), IL-10 (D1000B), IL-12 (D1200), IL-4 (D4050), IL-2 (D2050), IL-15 (DY247) and IFN- $\gamma$ (DIF50; All Bio-Rad Laboratories, Inc., Hercules, CA, USA) ELISA kits were used to determine serum levels of the inflammatory factors. The procedures were performed according to the manufacturer's protocols. The final results were recorded at $450 \mathrm{~nm}$ on an ELISA plate reader (Bio-Rad Laboratories, Inc.).

Small interfering RNA (siRNA) transfection. hPMCs were cultured to $80 \%$ confluence and transfected with siRNA that targeted TNF- $\alpha$ (si-TNF- $\alpha, 5$ '-UGGGGAACUCUUCCCUCU G-3') or si-vector containing scrambled siRNA (5'-CUCGUC UCAUUGATGACAGTT-3') using Lipofectamine ${ }^{\mathrm{TM}}$ RNAi MAX (Invitrogen; Thermo Fisher Scientific, Inc.) according to the manufacturer's protocol. A total of $100 \mathrm{pmol} \mathrm{si-TNF- \alpha}$ and si-vector (GenePharma Co., Ltd., Shanghai, China) were used for transfection. The subsequent experimentation was performed after $48 \mathrm{~h}$ transfection.

Western blot analysis. hPMCs were lysed in RIPA buffer (Sigma-Aldrich; Merck KGaA) containing a phosphatase inhibitor and protease inhibitor cocktail. Protein concentrations were determined by BCA protein assay kit (Pierce; Thermo Fisher Scientific, Inc.). Protein concentration was measured by a BCA protein assay kit (Thermo Scientific, Inc.). A total of $20 \mu \mathrm{g}$ protein extracts was subjected to $12.5 \%$ SDS-PAGE and then transferred to polyvinylidene membrane (EMD Millipore, Billerica, MA, USA). The primary antibodies of rat anti-human anti-TNF- $\alpha$ (1:1,000 dilution, ab667), anti-MMP-1 (1:1,000 dilution, ab137332), anti-MMP-9 (1:1,000 dilution, ab73734), high mobility group box-1 protein (HMGB1, 1:1,000 dilution, ab18256), ERK (1:2,000 dilution, ab196883, Abcam), pERK (1:2,000 dilution, ab214362, Abcam), AKT (1:1,000 dilution, ab8805, Abcam), pAKT (1:1,000 dilution, ab133458, Abcam), and $\beta$-actin (1:1,000 dilution, ab8226; all Abcam, Cambridge, UK) were used to incubate with the membranes for $120 \mathrm{~min}$ at $37^{\circ} \mathrm{C}$. Then goat anti-rabbit $\operatorname{IgG~mAb}(1: 5,000$ dilution, PV-6001, OriGene Technologies, Inc., Beijing, China) were added to the membranes for $60 \mathrm{~min}$ at $37^{\circ} \mathrm{C}$. Following this, the membrane was washed three times in TBST, and was developed using a chemiluminescence assay system (Roche Diagnostics, Basel, Switzerland) and exposed to Kodak exposure films. Densitometric quantification of the immunoblot data was performed by using the software of Quantity-One (version 3.23, Bio-Rad Laboratories, Inc.).

Histological assay. Lung specimens ( $\mathrm{n}=3$ in each group) obtained from patients and healthy volunteers as previously indicated (25). Specimens were prepared and fixed in $4 \%$ paraformaldehyde for $2 \mathrm{~h}$ at $37^{\circ} \mathrm{C}$. Paraffin-embedded tissue sections $(4 \mu \mathrm{m})$ were prepared and epitope retrieval was performed using Tris-HCl buffer for heat-induced epitope retrieval (AP-9005-050, Thermo Fisher Scientific, Inc.) for further analysis. The paraffin sections were quenched with hydrogen peroxide (3\%) for 10-15 $\mathrm{min}$, and subsequently blocked with a blocking solution $5 \%$ bovine serum albumin (Sigma-Aldrich; Merck $\mathrm{KGaA}$ ) for $10-15 \mathrm{~min}$ at $37^{\circ} \mathrm{C}$. Finally, the sections were incubated with goat anti-human anti-CD11b (1:1,000 dilution, ab133357, Abcam), anti-CD177 (1:1,000 dilution, ab203025, Abcam), or anti-CD31 (1:1,000 dilution, ab28364, Abcam) at $4^{\circ} \mathrm{C}$ for $12 \mathrm{~h}$. Sections were stained with the rabbit anti-goat horseradish peroxidase-conjugated anti-rabbit IgG (1:5,000 dilution, PV-6001, OriGene Technologies, Inc.) after washing with PBS three 
A
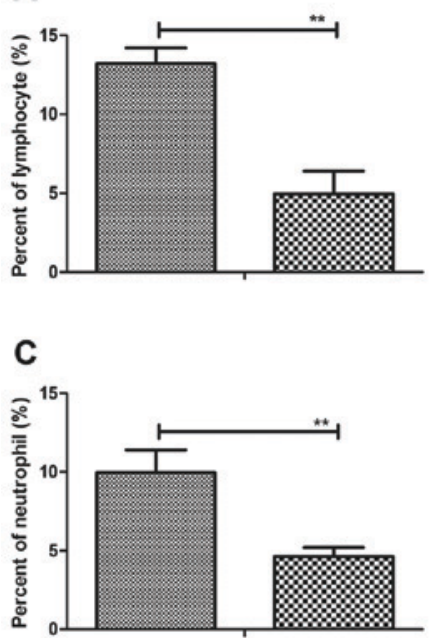

E

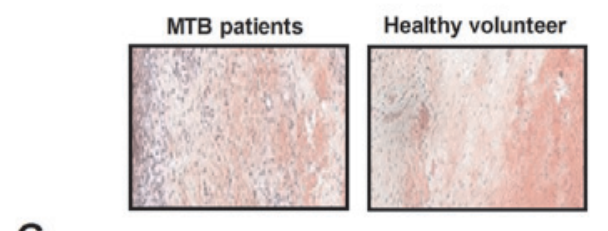

B
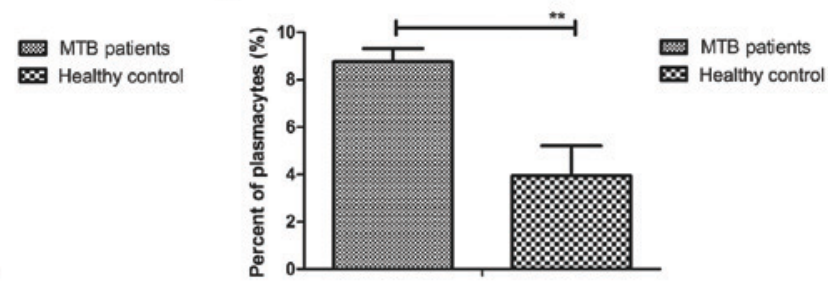

D

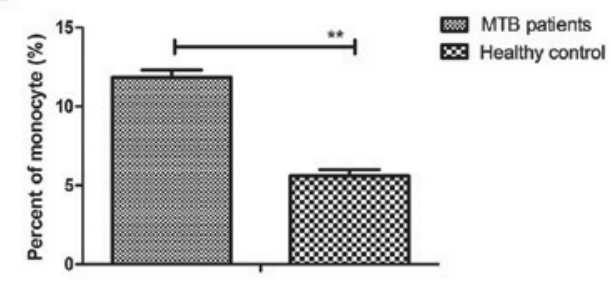

$\mathbf{F}$

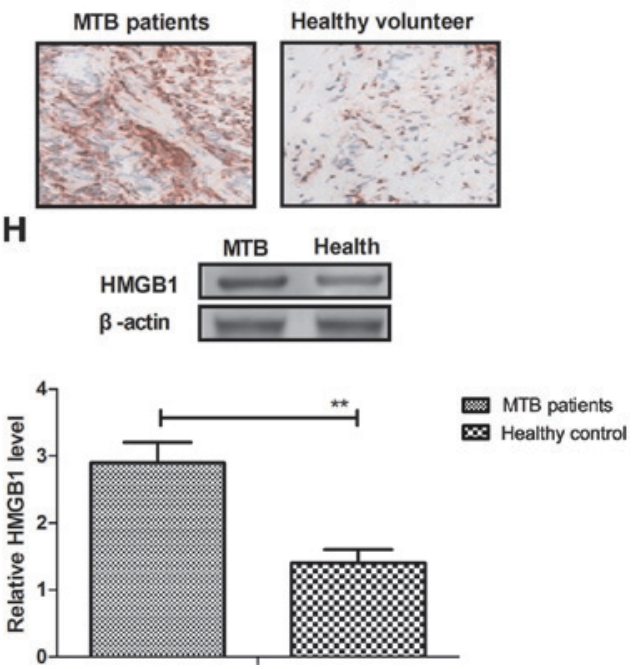

Figure 1. Changes in inflammatory cells in patients with MTB. The percentage of (A) lymphocytes, (B) plasmacytes, (C) neutrophils and (D) monocytes was analyzed in the serum of patients with MTB. The percentage of (E) macrophages, (F) mast cells and (G) endothelial cells was analyzed in the lung tissue of patients with MTB. (H) Expression levels of HMGB1 were analyzed in the lung tissue of patients with MTB. Data are expressed as the mean \pm standard deviation of three independent experiments. Magnification, $\mathrm{x} 40 .{ }^{* *} \mathrm{P}<0.01$ vs. healthy controls. HMGB1, high mobility group box-1; MTB, Mycobacterium tuberculosis infection.

times for $2 \mathrm{~h}$ at $37^{\circ} \mathrm{C}$. Visualization was achieved with peroxidase-labeled streptavidin-biotin and diaminobenzidine (DAB, Advansta, Inc., Menlo Park, CA, USA) for $~ 5$ min at $37^{\circ} \mathrm{C}$. The slides were examined with a Keyence Biozero BZ8100E microscope.

Flow cytometry. Serum was obtained as described above, and serum levels of lymphocytes, plasmacytes, neutrophils and monocytes in patients with pulmonary tuberculosis or healthy volunteers were analyzed using a flow cytometer. For detecting Th1 and Th2 cells, a Th1/Th2 (7 plex) Multiplex Immunoassay Kit (ab213389, Abcam) was used to measure the percentage of Th1 and Th2 cells, and TH1/Th2 ratio. All procedures were performed as previously described and the percentage of cells were analyzed using BD FACSCanto ${ }^{\mathrm{TM}}$ Software (version 2.0; BD Biosciences San Jose, CA, USA) as described previously (26).
Statistical analysis. Data are expressed as the mean \pm standard deviation. Significance was established with the SPSS 19.0 statistical (IBM Corp., Armonk, NY, USA) and GraphPad Prism 5 software (GraphPad Software, Inc., La Jolla, CA, USA). Comparisons between two groups were conducted by Student's t-test. $\mathrm{P}<0.05$ was considered to indicate a statistically significant difference.

\section{Results}

Analysis of inflammatory cells in serum in patients with M. tuberculosis infection. Clinical data from 124 patients with pulmonary tuberculosis revealed that immune cells were upregulated in the serum. As shown in Fig. 1A-D, the results indicated that serum concentration levels of lymphocytes, plasmacytes, neutrophils and monocytes were significantly increased in the patients with $M$. tuberculosis compared with 
A

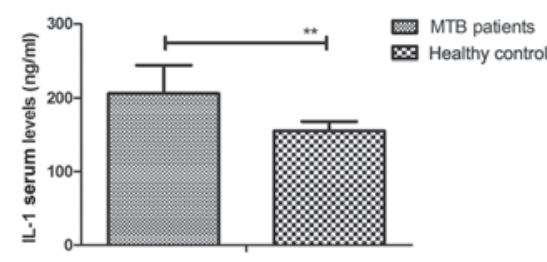

D

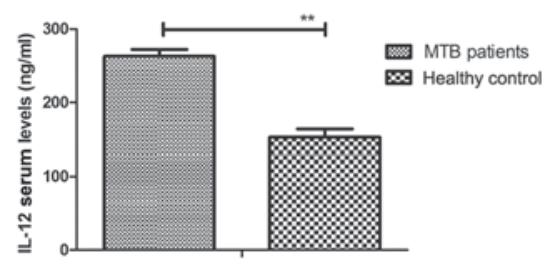

B

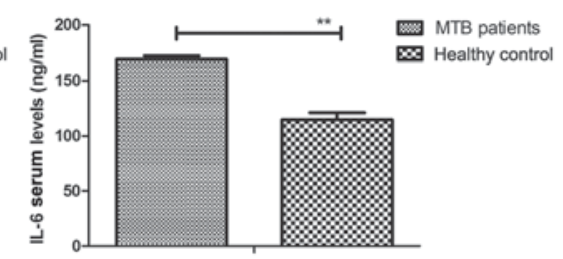

E

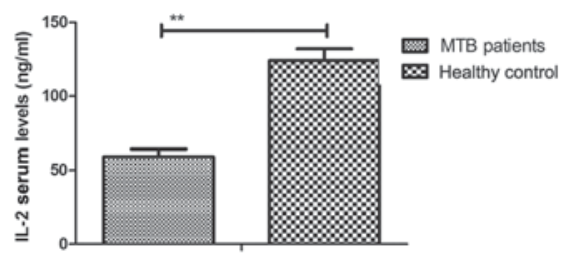

C

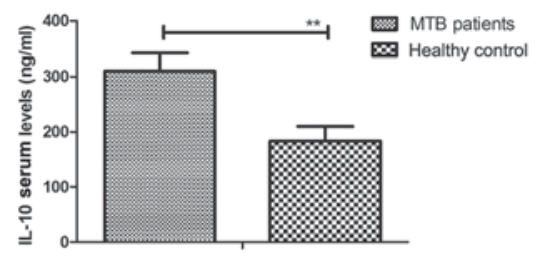

$\mathbf{F}$

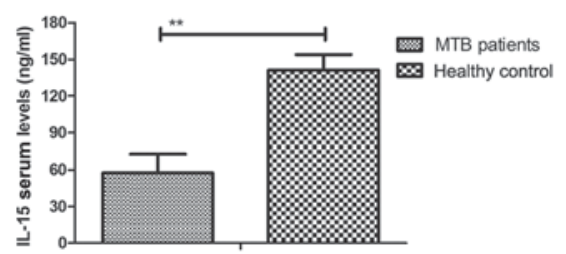

Figure 2. Inflammatory factors in patients with MTB. Serum levels of (A) IL-1, (B) IL-6, (C) IL-10, (D) IL-12, (E) IL-2 and (F) IL-15 were analyzed in patients with MTB. Data are expressed as the mean \pm standard deviation of three independent experiments. ${ }^{* *} \mathrm{P}<0.01$ vs. healthy controls. IL, interleukin; MTB, Mycobacterium tuberculosis infection.

healthy controls $(\mathrm{P}<0.01)$. In addition, outcomes indicated that macrophages, mast cells and endothelial cells were also increased in lung tissue in the patients with M. tuberculosis compared with healthy controls (Fig. 1E-G). Furthermore, it was observed that high HMGB1 expression levels were significantly upregulated in lung tissue in patients with M. tuberculosis compared with healthy controls $(\mathrm{P}<0.01$; Fig. $1 \mathrm{H}$ ), which would contribute to inflammation in patients. These outcomes suggest that inflammatory cells were upregulated in patients with M.tuberculosis.

Analysis of inflammatory factors in patients with $M$. tuberculosis. To detect the association between inflammatory factors and M. tuberculosis, serum levels of inflammatory factors were measured in patients with pulmonary tuberculosis in ICU, with healthy volunteers as the control. As shown in Fig. 2A-D, serum concentration levels of IL-1, IL-6, IL-10 and IL-12 were significantly upregulated in patients with pulmonary tuberculosis in ICU compared with healthy controls $(\mathrm{P}<0.01)$. However, significantly lower serum levels of IL-2 and IL-15 were observed in patients with pulmonary tuberculosis compared with healthy controls ( $\mathrm{P}<0.01$; Fig. $2 \mathrm{E}$ and F). These outcomes suggest that inflammatory factors were increased in the serum, while anti-inflammatory factors were decreased in patients with pulmonary tuberculosis in ICU.

Analysis of the balance of Th1/Th2 cytokines in patients with pulmonary tuberculosis. A previous study indicated that an imbalance of Th1/Th2 cytokines is a key indicator of inflammation and serves a critical function in the pathology of pulmonary tuberculosis in ICU (27). In the present study, the expression levels of Th1 and Th2 cytokines were measured in patients with pulmonary tuberculosis in ICU. As shown in Fig. 3A and B, significantly lower expression of Th1 cytokines and significantly higher expression of Th2 cytokines was observed in the serum of patients with pulmonary tuberculosis compared with healthy controls (both $\mathrm{P}<0.01$ ). In addition, the ratio of Th1/Th2 cytokines was significantly decreased in patients with pulmonary tuberculosis compared with healthy controls $(\mathrm{P}<0.01$; Fig. $3 \mathrm{C})$. In addition, significantly higher IL-4 and IL-17 levels (both $\mathrm{P}<0.01$ ) and significantly lower serum levels of IFN- $\gamma(\mathrm{P}<0.01)$ were observed (Fig. 3D-F), contributing to the imbalance of Th1/Th2 cytokines in patients with pulmonary tuberculosis compared with healthy controls. These outcomes suggest that an imbalance of Th1/Th2 cytokines is associated with pulmonary tuberculosis.

Analysis of TNF- $\alpha$ and MMP expression in hPMCs isolated from patients with pulmonary tuberculosis. It has previously been reported that expression levels of TNF- $\alpha$ and MMPs are correlated with the severity of patients with pulmonary tuberculosis $(28,29)$. In the present study, the levels of TNF- $\alpha$, MMP-1 and MMP-9 were evaluated in serum and hPMCs isolated from patients with pulmonary tuberculosis. As shown in Fig. 4A-C, the results indicated that serum levels of TNF- $\alpha$, MMP-1 and MMP-9 were significantly increased in patients with pulmonary tuberculosis compared with healthy controls (all $\mathrm{P}<0.01$ ). It was also observed that protein expression levels of TNF- $\alpha$, MMP-1 and MMP-9 were significantly upregulated in hPMCs isolated from patients with pulmonary tuberculosis (all $\mathrm{P}<0.01$; Fig. 4D-F). These results indicate that $M$. tuberculosis stimulates hPMCs to upregulate TNF- $\alpha$, MMP-1 and MMP-9, which may aggravate the severity of pulmonary tuberculosis.

Analysis of TNF- $\alpha$-induced ERK/Akt signaling pathway in hPMCs isolated from patients with pulmonary tuberculosis. To further analyze the molecular mechanism of TNF- $\alpha$, MMP-1 and MMP-9 upregulation in hPMCs, the ERK/Akt signaling pathway was evaluated in hPMCs isolated from patients with pulmonary tuberculosis. As shown in Fig. 5A and $\mathrm{B}$, the results indicated that expression levels of ERK and Akt were significantly upregulated in hPMCs isolated from patients with pulmonary tuberculosis compared with healthy controls (both $\mathrm{P}<0.01$ ). In addition, phosphorylation levels of ERK and Akt were significantly increased in hPMCs isolated from patients compared with healthy controls (both $\mathrm{P}<0.01$; 
A $\quad$ B

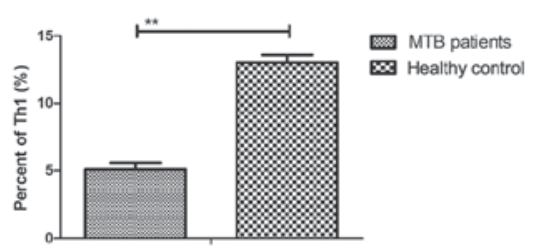

D

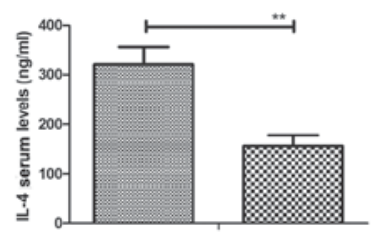

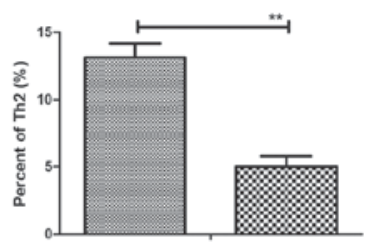

E

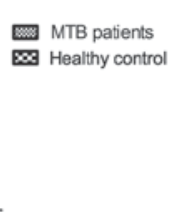

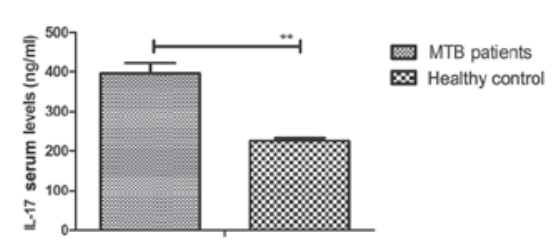

C

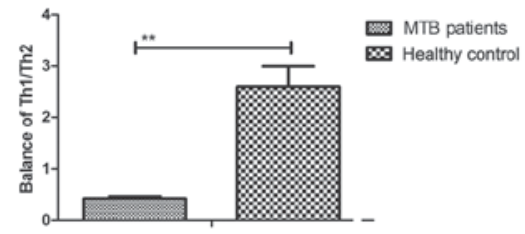

$\mathbf{F}$

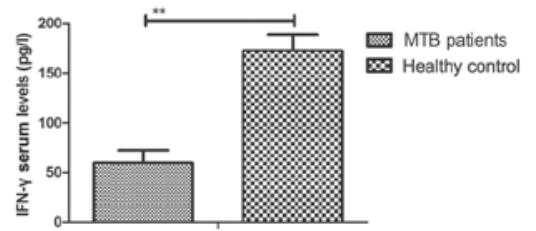

Figure 3. Evaluation of Th1/Th2 cytokines in patients with MTB. Serum levels of (A) Th1 and (B) Th2 cells were evaluated in patients with MTB. (C) The balance of Th1/Th2 cytokines was analyzed in patients with MTB. Serum levels of (D) IL-4, (E) IL-17 and (F) IFN- $\gamma$ were analyzed in patients with MTB Data are expressed as the mean \pm standard deviation of three independent experiments. ${ }^{* *} \mathrm{P}<0.01$ vs. healthy controls. Th, T helper cell; IL, interleukin; IFN, interferon; MTB, Mycobacterium tuberculosis infection.

A

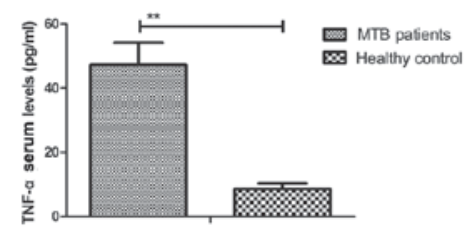

D

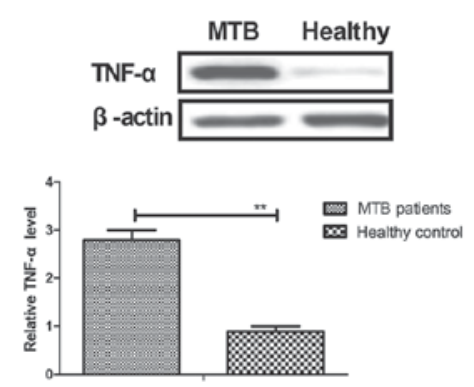

B

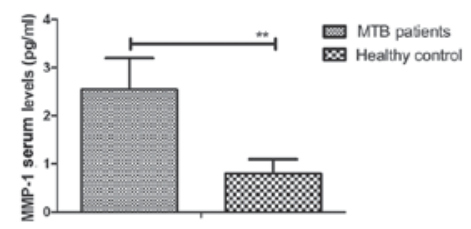

$\mathbf{E}$
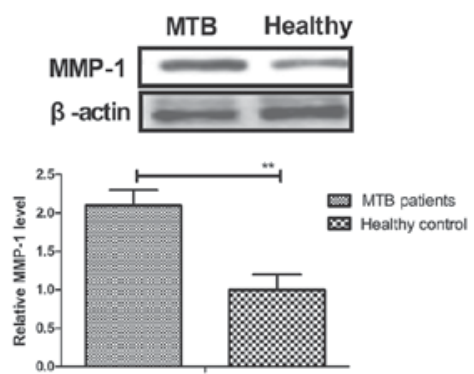

C

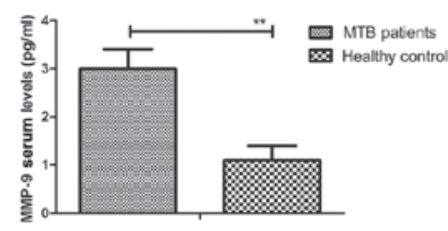

$\mathbf{F}$
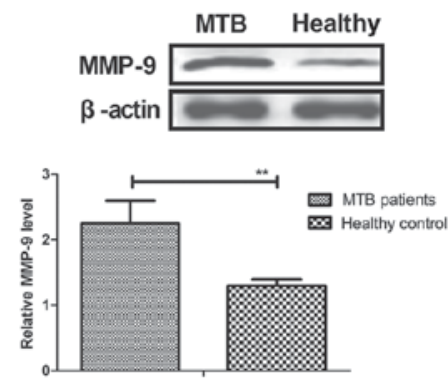

Figure 4. Expression levels of TNF- $\alpha$ and MMPs in patients with MTB. Serum levels of (A) TNF- $\alpha$, (B) MMP-1 and (C) MMP-9 were evaluated in patients with MTB. Protein expression levels of (D) TNF- $\alpha$, (E) MMP-1 and (F) MMP-9 in human pleural mesothelial cells isolated from patients with MTB. Data are expressed as the mean \pm standard deviation of three independent experiments. ${ }^{* *} \mathrm{P}<0.01$ vs. healthy controls. TNF, tumor necrosis factor; MMP, matrix metalloproteinase; MTB, Mycobacterium tuberculosis infection.

Fig. 5C and D). Furthermore, it was identified that inhibition of TNF- $\alpha$ expression suppressed ERK and Akt expression and phosphorylation (Fig. 5E and F). MMP-1 and MMP-9 expression levels were also significantly downregulated by TNF- $\alpha$ knockdown in hPMCs $(\mathrm{P}<0.01$; Fig. $5 \mathrm{G}$ and $\mathrm{H})$. These results suggest that TNF- $\alpha$ mediates the upregulation of MMP-1 and MMP-9 expression through the ERK/Akt signaling pathway in hPMCs in patients with pulmonary tuberculosis.

\section{Discussion}

Pulmonary tuberculosis is a chronic infectious disease, which is characterized by lung granulomatous lesion formation and severe inflammatory responses $(30,31)$. A previous study suggested that inflammatory factors induced by inflammatory responses in lung tissue contributed to the severity of pulmonary tuberculosis when patients were infected with M. tuberculosis (32). Clinically, the activity of pulmonary tuberculosis inflammation caused by Mycobacterium has been identified as an indicator for patients' disease severity and drug tolerance (33). In the present study, inflammatory responses were investigated in patients with pulmonary tuberculosis in ICU. Serum levels of inflammatory cells, including lymphocytes, plasmacytes, neutrophils and monocytes were studied in patients with pulmonary tuberculosis in ICU. It was identified that inflammatory responses are 
A

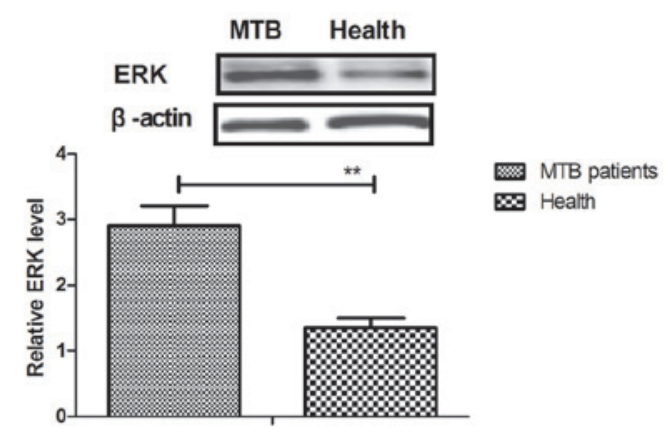

C
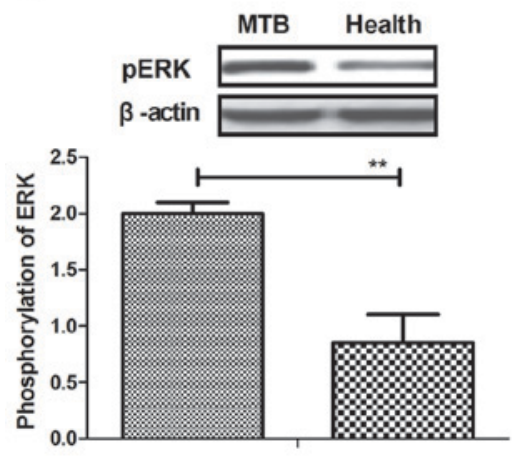

E
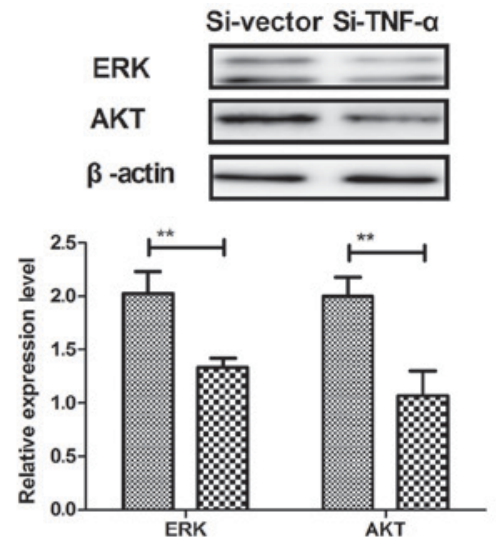

G

\section{Si-vector Si-TNF-a}
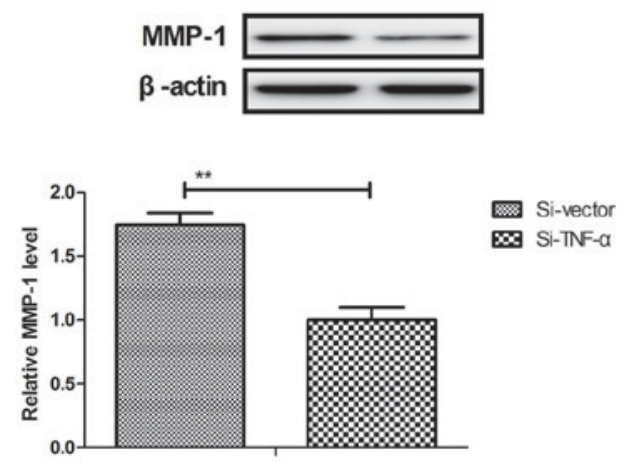

B

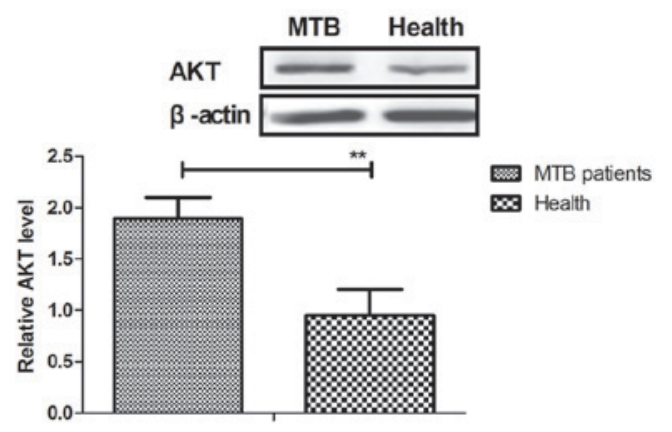

D
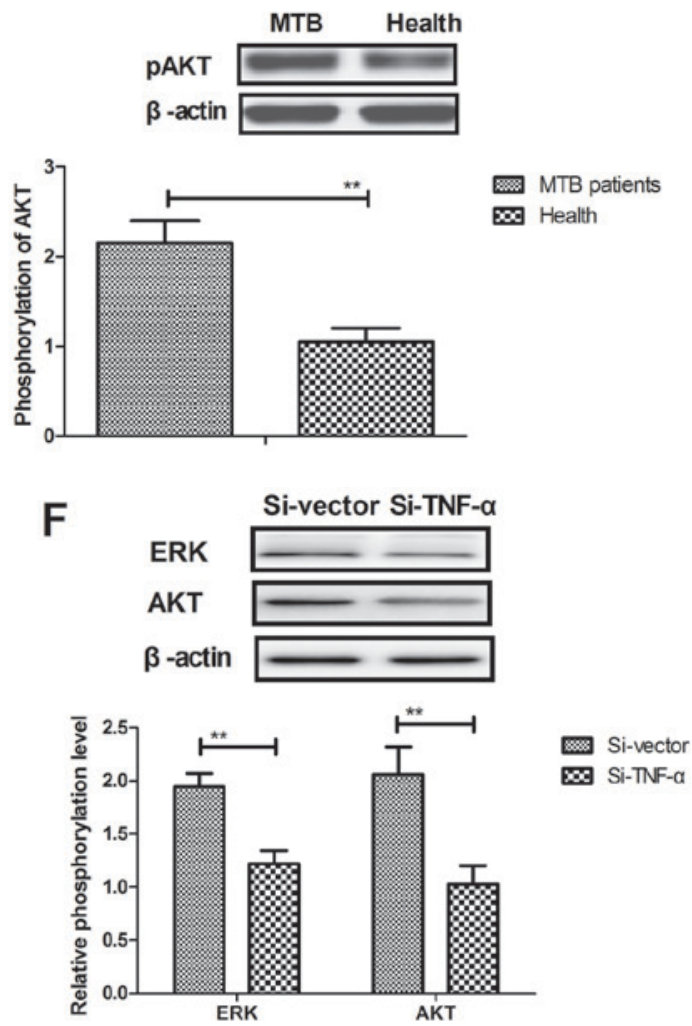

$\mathrm{H}$
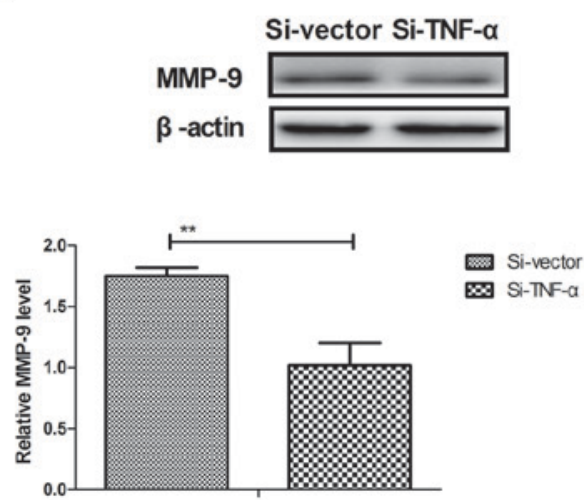

Figure 5. Analysis of TNF- $\alpha$-induced ERK/ATK signaling pathway in hPMCs isolated from patients with MTB. Expression levels of (A) ERK and (B) Akt were evaluated in hPMCs isolated from patients with MTB. Phosphorylation levels of (C) ERK and (D) Akt in hPMCs isolated from patients with MTB. (E) Expression levels and (F) phosphorylation levels of ERK and Akt were evaluated in si-TNF- $\alpha$-treated hPMCs. Expression levels of (G) MMP-1 and (H) MMP-9 were evaluated in si-TNF- $\alpha$-treated hPMCs. Data are expressed as the mean \pm standard deviation of three independent experiments. ${ }^{* *} \mathrm{P}<0.01$ vs. healthy controls. TNF, tumor necrosis factor; MMP, matrix metalloproteinase; ERK, extracellular-signal-regulated kinase; hPMC, human pleural mesothelial cell; MTB, Mycobacterium tuberculosis infection; si, small interfering RNA. 
enhanced and inflammatory factors are stimulated in patients with $M$. tuberculosis infection. Notably, it was observed that the balance of Th1/Th2 cytokines was disturbed in patients with pulmonary tuberculosis compared with healthy controls. It was observed that pulmonary tuberculosis upregulated TNF- $\alpha$ expression through the ERK/Akt signaling pathway in hPMCs.

Inflammatory cells are the most common pathological characteristics for patients with pulmonary tuberculosis infected with Mycobacterium (34). These inflammatory cells include lymphocytes, plasmacytes, neutrophils, monocytes, macrophages, mast cells and endothelial cells. Previous studies have demonstrated that the morphology and function of plasmacytes are associated with the progression of tuberculosis $(35,36)$. In addition, Iliaz et al (37) indicated that neutrophil/lymphocyte ratio could be used as a reference in the differential diagnosis of tuberculosis. Furthermore, previous investigations have suggested that suppression of $M$.tuberculosis growth can upregulate TNF- $\alpha$, which increases the levels of human monocytes in patients with pulmonary tuberculosis $(38,39)$. These reports suggest that inhibition of inflammatory cells may be a potential target for the treatment of pulmonary tuberculosis. The present results indicated that inflammatory cells were increased in the serum of patients with pulmonary tuberculosis.

Inflammatory factors secreted by inflammatory cells are reported to be associated with pathogenicity and severity of pulmonary tuberculosis, and may induce other tuberculosis-related diseases (40). In the present study, the expression levels of IL-1, IL-6, IL-10 and IL-12 were investigated in patients with pulmonary tuberculosis. Katti (41) assessed serum IL-1, IL-2 and IFN- $\gamma$ levels in patients with pulmonary tuberculosis and reported that increased IL-1 is an indicator for Th1 responses. In the present study, it was observed that IL-1 serum levels were upregulated in patients with pulmonary tuberculosis. Serum levels of IL-6 act as a potential biomarker of disease progression in pulmonary tuberculosis following anti-tuberculosis drug therapy (42). In the present study, IL-6 serum levels were increased in patients compared with the controls. The present results also indicated that serum levels of IL-2 and IL-15 were decreased in the serum of patients with pulmonary tuberculosis, which may contribute to understanding the molecular mechanism of inflammatory responses in pulmonary tuberculosis. Furthermore, expression levels and polymorphisms of IL-10 and TNF- $\alpha$ are affected in patients with pulmonary tuberculosis (43). In the present study, it was demonstrated that serum levels of IL-10 and TNF- $\alpha$ were increased in patients with pulmonary tuberculosis compared with healthy controls, which may lead to inflammatory responses in patients.

Understanding the mechanisms of pulmonary tuberculosis is beneficial for preventing and treating M. tuberculosis infection (44). In the present study, the ERK/Akt signaling pathway was analyzed in hPMCs isolated from patients with $M$. tuberculosis infection. The results indicated that $M$. tuberculosis induced higher levels of TNF- $\alpha$, mediated by the ERK/Akt signal pathway, resulting in upregulation of MMP-1 and MMP-9 expression in hPMCs. Although MMP-9 activity has been characterized in processes of granuloma formation in pleural tuberculosis, to the best of our knowledge, the signal pathway in inflammatory responses in tuberculosis pleural disease has not yet been reported (45). The present investigations have elaborated on the molecular mechanism of MMP-9 upregulation and indicated that TNF- $\alpha$-induced upregulation of MMP-1 and MMP-9 expression is mediated by the ERK/Akt signaling pathway in hPMCs in patients with pulmonary tuberculosis.

In conclusion, the present study indicates that inflammatory responses and inflammatory factors are upregulated in patients with pulmonary tuberculosis. Notably, the present findings suggest that M. tuberculosis-induced inflammatory responses and factors are mediated by MMP-1 and MMP-9 expression via the TNF- $\alpha$-mediated ERK/Akt signaling pathway in hPMCs. These results suggest that inhibition of inflammatory responses and inflammatory factors may be beneficial for the treatment of patients with pulmonary tuberculosis in ICU.

\section{Acknowledgements}

This study was supported by the Youth Programme of Beijing Municipal Administration of Hospitals (grant no. QML20151501) and the National Science and Technology Major Project of China (grant nos. 2015ZX10004801-003 and 2016ZX10003001-011).

\section{References}

1. Mahmoud ES, Baharoon SA, Alsafi E and Al-Jahdaly H: Acute respiratory distress syndrome complicating community-acquired pneumonia secondary to mycobacterium tuberculosis in a tertiary care center in Saudi Arabia. Saudi Med J 37: 973-978, 2016.

2. Sood S, Yadav A and Shrivastava R: Mycobacterium aurum is Unable to Survive Mycobacterium tuberculosis latency associated stress conditions: Implications as non-suitable model organism. Indian J Microbiol 56: 198-204, 2016.

3. WHO: Tuberculosis. http://www.who.int/mediacentre/factsheets/ fs104/en/. Updated October 2017.

4. Maruri F, Sterling TR, Kaiga AW, Blackman A, van der Heijden YF, Mayer C, Cambau E and Aubry A: A systematic review of gyrase mutations associated with fluoroquinolone-resistant Mycobacterium tuberculosis and a proposed gyrase numbering system. J Antimicrob Chemother 67: 819-831, 2012.

5. Georghiou SB, Magana M, Garfein RS, Catanzaro DG, Catanzaro A and Rodwell TC: Evaluation of genetic mutations associated with Mycobacterium tuberculosis resistance to amikacin, kanamycin and capreomycin: A systematic review. PLoS One 7: e33275, 2012.

6. Phelippeau M and Petureau F: Severe pulmonary tuberculosis in the ICU, diagnosis and treatment. Rev Pneumol Clin 71: 294-296, 2015 (In French).

7. Silva DR, Gazzana MB and Dalcin Pde T: Severe tuberculosis requiring ICU admission. J Bras Pneumol 38: 386-394, 2012.

8. Luo H, Pang L and Xie J: Biosynthesis and regulation of mycolic acids in Mycobacterium tuberculosis-a review. Wei Sheng Wu Xue Bao 52: 146-151, 2012.

9. Chiappini E, Bonsignori F, Accetta G, Boddi V, Galli L, Biggeri A and De Martino M: Interferon-gamma release assays for the diagnosis of Mycobacterium tuberculosis infection in children: A literature review. Int J Immunopathol Pharmacol 25: 335-343, 2012.

10. Chiappini E, Accetta G, Bonsignori F, et al: Interferon-gamma release assays for the diagnosis of Mycobacterium tuberculosis infection in children: a systematic review and meta-analysis. Int J Immunopathol Pharmacol 25: 557-564, 2012.

11. Lamrabet $\mathrm{O}$ and Drancourt $\mathrm{M}$ : Genetic engineering of Mycobacterium tuberculosis: A review. Tuberculosis (Edinb) 92: 365-376, 2012. 
12. Raslan WF, Rabaan A and Al-Tawfiq JA: The predictive value of Gen-Probe's amplified Mycobacterium tuberculosis direct test compared with culturing in paraffin-embedded lymph node tissue exhibiting granulomatous inflammation and negative acid fast stain. J Infect Public Health 7: 251-256, 2014.

13. Gunluoglu G, Yazar EE, Veske NS, Seyhan EC and Altin S: Mean platelet volume as an inflammation marker in active pulmonary tuberculosis. Multidiscip Respir Med 9: 11, 2014.

14. Sahin O and Ziaei A: The role of methotrexate in resolving ocular inflammation after specific therapy for presumed latent syphilitic uveitis and presumed tuberculosis-related uveitis. Retina 34: 1451-1459, 2014.

15. Dorhoi A and Kaufmann SH: Perspectives on host adaptation in response to Mycobacterium tuberculosis: Modulation of inflammation. Semin Immunol 26: 533-542, 2014.

16. Zumla A, Rao M, Parida SK, Keshavjee S, Cassell G, Wallis R, Axelsson-Robertsson R, Doherty M, Andersson J and Maeurer M: Inflammation and tuberculosis: Host-directed therapies. J Intern Med 277: 373-387, 2015.

17. Tsenova L, O'Brien P, Holloway J, Peixoto B, Soteropoulos P, Fallows D, Kaplan G and Subbian S: Etanercept exacerbates inflammation and pathology in a rabbit model of active pulmonary tuberculosis. J Interferon Cytokine Res 34: 716-726, 2014.

18. Caspers L, Makhoul D, Ebraert H, Michel O and Willermain F: Clinical manifestations of patients with intraocular inflammation and positive QuantiFERON-TB gold in-tube test in a country nonendemic for tuberculosis. Am J Ophthalmol 158: 646-647, 2014.

19. Aranday-Cortes E, Hogarth PJ, Kaveh DA, Whelan AO Villarreal-Ramos B, Lalvani A and Vordermeier HM: Transcriptional profiling of disease-induced host responses in bovine tuberculosis and the identification of potential diagnostic biomarkers. PLoS One 7: e30626, 2012.

20. Chen WL, Sheu JR, Chen RJ, Hsiao SH, Hsiao CJ, Chou YC, Chung CL and Hsiao G: Mycobacterium tuberculosis Upregulates TNF- $\alpha$ Expression via TLR2/ERK signaling and induces MMP-1 and MMP-9 production in human pleural mesothelial cells. PLoS One 10: e0137979, 2015

21. Mayordomo L, Marenco JL, Gomez-Mateos J and Rejon E: Pulmonary miliary tuberculosis in a patient with anti-TNF-alpha treatment. Scand J Rheumatol 31: 44-45, 2002.

22. Mihăltan F: TNF-alpha blockers, rheumatoid arthritis and pulmonary tuberculosis. Pneumologia 56: 212-216, 2007.

23. Wang $\mathrm{CH}$, Lin HC, Lin SM, Huang CD, Liu CY, Huang $\mathrm{KH}$ Hsieh LL, Chung KF and Kuo HP: MMP-1(-1607G) polymorphism as a risk factor for fibrosis after pulmonary tuberculosis in Taiwan. Int J Tuberc Lung Dis 14: 627-634, 2010.

24. Ong CW, Elkington PT, Brilha S, Ugarte-Gil C, Tome-Esteban MT, Tezera LB, Pabisiak PJ, Moores RC, Sathyamoorthy T, Patel V, et al: Neutrophil-Derived MMP-8 drives AMPK-dependent matrix destruction in human pulmonary tuberculosis. PLoS Pathog 11: e1004917, 2015.

25. Nowak K, Hanusch C, Kölbel HC, Schwarzbach M, Post S Beck G, Gebhard MM, Metzger R and Hohenberger P: Alterations of tumor and normal tissue of human lung cancer resection specimens after isolation perfusion. J Physiol Pharmacol 58 (Suppl 5): 501-511, 2007.

26. Bajnok A, Kaposi A, Kovacs L, Vasarhelyi B, Balog A and Toldi G: Analysis by flow cytometry of calcium influx kinetics in peripheral lymphocytes of patients with rheumatoid arthritis. Cytometry A 83: 287-293, 2013.

27. Wang L, Cai Y, Cheng Q,Hu Y and Xiao H: Imbalance of Th1/Th2 cytokines in patients with pulmonary tuberculosis. Zhonghua Jie $\mathrm{He} \mathrm{He} \mathrm{Hu}$ Xi Za Zhi 25: 535-537, 2002 (In Chinese).

28. Cavalcanti YV, Brelaz MC, Neves JK, Ferraz JC and Pereira VR Role of TNF-Alpha, IFN-Gamma and IL-10 in the development of pulmonary tuberculosis. Pulm Med 2012: 745483, 2012.

29. Anand SP and Selvaraj P: Effect of 1, 25 dihydroxyvitamin $D(3)$ on matrix metalloproteinases MMP-7, MMP-9 and the inhibitor TIMP-1 in pulmonary tuberculosis. Clin Immunol 133: 126-131, 2009.

30. Gonzalez-Angulo Y, Wiysonge CS, Geldenhuys H, Hanekom W, Mahomed H, Hussey G and Hatherill M: Sputum induction for the diagnosis of pulmonary tuberculosis: A systematic review and meta-analysis. Eur J Clin Microbiol Infect Dis 31: 1619-1630, 2012.
31. Badyal RK, Kataria AS, Sachdeva K and Kapoor S: Macrolithiasis in pulmonary tuberculosis: An autopsy report with review of literature. Indian J Pathol Microbiol 55: 119-120, 2012.

32. Davis JL, Cattamanchi A, Cuevas LE, Hopewell PC and Steingart KR: Diagnostic accuracy of same-day microscopy versus standard microscopy for pulmonary tuberculosis: A systematic review and meta-analysis. Lancet Infect Dis 13: 147-154, 2013.

33. Elipashev AA, Nikol'skii VO and Shprykov AS: Prognostic value of morphological signs of the activity of tuberculous inflammation in patients with circumscribed drug-resistant pulmonary tuberculosis. Arkh Patol 72: 40-43, 2010 (In Russian).

34. Kaminskaia GO, Popov EV and Romanov VV: Comparison of systemic manifestations of inflammation in torpid pulmonary tuberculosis and respiratory sarcoidosis. Probl Tuberk Bolezn Legk: 25-29, 2008.

35. Skogmar S, Schön T, Balcha TT, Sturegard E, Jansson M and Björkman P: Plasma levels of neopterin and C-reactive protein (CRP) in tuberculosis (TB) with and without HIV coinfection in relation to CD4 cell count. PLoS One 10: e0144292, 2015.

36. Koguchi Y, Kawakami K, Uezu K, Fukushima K, Kon S, Maeda M, Nakamoto A, Owan I, Kuba M, Kudeken N, et al: High plasma osteopontin level and its relationship with interleukin-12-mediated type $1 \mathrm{~T}$ helper cell response in tuberculosis. Am J Respir Crit Care Med 167: 1355-1359, 2003.

37. Iliaz S, Iliaz R, Ortakoylu G, Bahadir A, Bagci BA and Caglar E: Value of neutrophil/lymphocyte ratio in the differential diagnosis of sarcoidosis and tuberculosis. Ann Thorac Med 9: 232-235, 2014.

38. Azfar SF and Islam N: Suppression of mycobacterium tuberculosis induced reactive oxygen species and tumor necrosis factor-alpha activity in human monocytes of systemic lupus erythematosus patients by reduced glutathione. Oman Med J 27: $11-19,2012$.

39. Kim K, Sohn H, Kim JS, Choi HG, Byun EH, Lee KI, Shin SJ, Song CH, Park JK and Kim HJ: Mycobacterium tuberculosis Rv0652 stimulates production of tumour necrosis factor and monocytes chemoattractant protein-1 in macrophages through the Toll-like receptor 4 pathway. Immunology 136: 231-240, 2012.

40. Worodria W, Menten J, Massinga-Loembe M, Mazakpwe D, Bagenda D, Koole O, Mayanja-Kizza H, Kestens L, Mugerwa R, Reiss P, et al: Clinical spectrum, risk factors and outcome of immune reconstitution inflammatory syndrome in patients with tuberculosis-HIV coinfection. Antivir Ther 17: 841-848, 2012.

41. Katti MK: Assessment of serum IL-1, IL-2 and IFN- $\gamma$ levels in untreated pulmonary tuberculosis patients: Role in pathogenesis. Arch Med Res 42: 199-201, 2011.

42. Chowdhury IH, Choudhuri S, Sen A, Bhattacharya B, Ahmed AM, Hazra A, Pal NK and Bahar B: Serum interleukin 6 (IL-6) as a potential biomarker of disease progression in active pulmonary tuberculosis following anti-tuberculosis drug therapy. Mol Immunol 63: 601-602, 2015.

43. Scola L, Crivello A, Marino V, Gioia V, Serauto A, Candore G, Colonna-Romano G, Caruso C and Lio D: IL-10 and TNF-alpha polymorphisms in a sample of Sicilian patients affected by tuberculosis: Implication for ageing and life span expectancy. Mech Ageing Dev 124: 569-572, 2003

44. Erdemli SB, Gupta R, Bishai WR, Lamichhane G, Amzel LM and Bianchet MA: Targeting the cell wall of Mycobacterium tuberculosis: Structure and mechanism of L, D-transpeptidase 2. Structure 20: 2103-2115, 2012.

45. Sheen P, O'Kane CM, Chaudhary K, Tovar M, Santillan C, Sosa J, Caviedes L, Gilman RH, Stamp G and Friedland JS: High MMP-9 activity characterises pleural tuberculosis correlating with granuloma formation. Eur Respir J 33: 134-141, 2009.

This work is licensed under a Creative Commons Attribution-NonCommercial-NoDerivatives 4.0 International (CC BY-NC-ND 4.0) License. 